Published in final edited form as:

Stroke. 2015 August ; 46(8): 2063-2068 doi:10.1

\title{
Meta-analysis of genome-wide association studies identifies
}

Cara L. Carty, PhD ", Keith L. Keene, PhD ", Yu-Ching Cheng, PhD , James F. Meschia, D' ${ }^{5}$ Wei-Min Chen, PhD hen S. Rich, $\mathrm{PhD}^{6}$, Salman Tajuddin, $\mathrm{MD}, \mathrm{PhD}^{7}$, Alan B. Zonderman, $\mathrm{PhD}^{7}$ Michele K. Evans, $\mathrm{MD}^{7}$, Carl D. Langefeld, $\mathrm{PhD}^{9}$, Rebecca Gottesman, MD, $\mathrm{PhD}^{10}$, Thomas . Mosley, PhD ${ }^{11}$, Eyal Shahar, MD, MPH ${ }^{12}$, Daniel Woo, MD ${ }^{13}$, Kristine Yaffe, MD ${ }^{14}$ YongMei Liu, MD, PhD, Michèle M. Sale, PhD', Martin Dichgans, MD ${ }^{15}$, Rainer Malik, hD ${ }^{8,16}$, Charles Kooperberg, PhD $^{1}$, Alexander Reiner, MD, MS ${ }^{1,8}$, Bradford B. Worrall, MD, Consortia

${ }^{3}$ Baltimore Veterans Administration Medical Center, Baltimore, MD

University of Maryland School of Medicine, Baltimore, MD

National Institute of Aging, $\mathrm{NIH}$, Bethesda, MD

University of Washington, Seattle, WA

Wake Forest University, Winston-Salem, NC

Johns Hopkins University School of Medicine, Baltimore, MD

1 University of Mississippi Medical Center, Jackson, MS

University of Arizona, Tucson, AZ

University of Cincinnati, Cincinnati, OH

Cooperative, Seattle, WA

Corresponding author: Cara L. Carty, Center for Translational Science, George Washington University, Children's National Medical Center, 111 Michigan Ave NW, Washington, DC 20010, Office: 202-476-3252, Fax: 202-476-3425, ccarty@cnmc.org. authors contributed equally to this work

DISCLOSURES: BBW is an associate editor for the journal Neurology 
${ }^{17}$ University of Texas, Houston, TX

\section{Abstract}

Background and Purpose-The majority of genome-wide association studies (GWAS) of stroke have focused on European-ancestry populations; however, none has been conducted in African-Americans despite the disproportionately high burden of stroke in this population. The Consortium of Minority Population genome-wide Association Studies of Stroke (COMPASS) was established to identify stroke susceptibility loci in minority populations.

Methods-Using METAL, we conducted meta-analyses of GWAS in 14,746 African-Americans (1,365 ischemic and 1,592 total stroke cases) from COMPASS, and tested SNPs with $\mathrm{P}<10^{-6}$ for validation in METASTROKE, a consortium of ischemic stroke genetic studies in Europeanancestry populations. We also evaluated stroke loci previously identified in European-ancestry populations.

Results-The 15q21.3 locus linked with lipid levels and hypertension was associated with total stroke (rs4471613, $\left.\mathrm{P}=3.9 \times 10^{-8}\right)$ in African-Americans. Nominal associations $\left(\mathrm{P}<10^{-6}\right)$ for total or ischemic stroke were observed for 18 variants in or near genes implicated in cell cycle/ mRNA pre-splicing ( $P T P R G, C D C 5 L)$, platelet function (HPS4), blood-brain barrier permeability (CLDN17), immune response (ELTD1, WDFY4, ILIF10-ILIRN), and histone modification (HDAC9). Two of these loci achieved nominal significance in METASTROKE: 5q35.2 ( $\mathrm{P}=0.03)$, and 1p31.1 ( $\mathrm{P}=0.018)$. Four of 7 previously reported ischemic stroke loci (PITX2, HDAC9, $C D K N 2 A / C D K N 2 B$ and $Z F H X 3)$ were nominally associated $(\mathrm{P}<0.05)$ with stroke in COMPASS.

Conclusions-We identified a novel SNP associated with total stroke in African-Americans and found that ischemic stroke loci identified in European-ancestry populations may also be relevant for African-Americans. Our findings support investigation of diverse populations to identify and characterize genetic risk factors, and the importance of shared genetic risk across populations.

\section{Keywords}

stroke; GWAS; genetic association; African American; meta-analysis

\section{INTRODUCTION}

As the fourth leading cause of death and a leading cause of long-term disability, stroke causes a substantial burden of mortality and morbidity in the United States $1^{1}$. Stroke is a heterogeneous disease consisting of multiple subtypes, each with unique etiologies ${ }^{2}$ and risk factors ${ }^{3}$. Familial aggregation studies suggest that stroke has a substantial genetic component ${ }^{4}, 5$. Recent genome-wide association studies (GWAS), conducted almost exclusively in individuals of European-ancestry, have identified stroke susceptibility loci on chromosomes 4q25 $, 7 \mathrm{p} 21.1^{7}, 6 \mathrm{p} 21^{8}, 9 \mathrm{p} 21^{6}, 11 \mathrm{q} 22^{9}, 12 \mathrm{p} 13^{10}, 12 \mathrm{q} 24^{11}$, and 16q22 $2^{12}$.

However, stroke incidence and mortality in African Americans are both nearly twice that in European- Americans. ${ }^{13,14}$ Moreover, African-Americans suffer strokes at younger ages on average, and more frequently endure post-stroke disability ${ }^{13}$. 
Using data obtained from the newly-formed Consortium of Minority Population genomewide Association Studies of Stroke (COMPASS), we conducted the first discovery GWAS meta-analysis of stroke in African-Americans, validated our findings in the large METASTROKE consortium of ischemic stroke genetic studies in European-ancestry populations ${ }^{12}$, and determined if GWAS findings robustly associated with ischemic stroke in European-ancestry populations were also associated with stroke in African-Americans.

\section{METHODS}

\section{Study population}

African-American individuals with physician-adjudicated stroke and genome-wide single nucleotide polymorphism (SNP) data were included in these analyses. COMPASS includes cohort studies-Atherosclerosis Risk in Communities (ARIC) Study ${ }^{15}$, Cardiovascular Health Study (CHS) ${ }^{16}$, Dynamics of Health, Aging and Body Composition Study $(\mathrm{HABC})^{17}$, and the Women's Health Initiative $(\mathrm{WHI})^{18}$,-and case-control studies-Genetics of Early Onset Stroke (GEOS) ${ }^{19}$, Ischemic Stroke Genetics Study (ISGS) ${ }^{20}$, Vitamin Intervention for Stroke Prevention (VISP) ${ }^{21}$ and Healthy Aging in Neighborhoods of Diversity across the Life Span (HANDLS) ${ }^{22}$, -as well as an affected sibpair study-Siblings with Ischemic Stroke Study (SWISS) ${ }^{23}$.

\section{Outcomes}

Adjudicated ischemic or total strokes from all studies excluding HANDLS were analyzed. Race/ethnicity- and sex-matched controls were randomly selected from HANDLS and used as controls in the analyses of SWISS-ISGS and VISP, which lacked genotyped controls. All studies provided data for the total stroke analysis which included ischemic and hemorrhagic strokes but excluded subarachnoid hemorrhage. All studies contributed to the ischemic stroke analyses with the exception of HABC, which lacked stroke subtype information. In the cohort studies, only first (incident) clinically validated strokes were considered and individuals with baseline history of stroke were excluded.

\section{Genotype data}

All studies imputed SNPs using HapMap II reference populations; SNPs with low imputation quality $\left(\mathrm{r}^{2}<0.3\right)$ were excluded. We analyzed SNPs available in two or more studies with minor allele frequency (MAF) $\searrow 0.01$ and passing stringent quality control criteria, for a total of $\sim 2.6$ million SNPs. The Supplement contains additional details regarding study designs, stroke definition, adjudication procedures and genotyping.

\section{Analysis}

We used additive genetic models with a count of variant alleles $(0,1$, or 2$)$ for each genotyped SNP or allelic dose for imputed SNPs. Cohort studies used Cox proportional hazards models to evaluate associations between SNPs and the time to incident stroke. Casecontrol studies used logistic regression models (additional details in Supplement). To control for potential population stratification, principal components of global ancestry were estimated in each study and included as covariates. Models were additionally adjusted, as appropriate, for age, sex and site. In each study, the distribution of test statistics was 
reviewed using Q-Q plots to detect potential inflation due to population stratification; no large deviations were noted. We combined study-specific results in fixed effects metaanalyses with inverse variance weighting using METAL; $;{ }^{24} \mathrm{P}$-value meta-analyses were also conducted. The genome-wide significance threshold was $\mathrm{P}<5 \times 10^{-8}$ for the GWAS discovery but we investigated all SNPs with $\mathrm{P}<10^{-6}$.

\section{Validation of COMPASS Findings}

Due to the absence of another large African-American sample with GWAS and adjudicated stroke data, we performed a 'look-up' of COMPASS SNPs with $\mathrm{P}<10^{-6}$ in the METASTROKE ischemic stroke results. Given the known differences in linkage disequilibrium (LD) patterns between populations of European- and African-ancestry, we expanded the region of interest for each locus to include available SNPs $\pm 500 \mathrm{~kb}$ of the index SNPs. We applied Bonferroni correction to account for the number of loci tested.

As a secondary aim, we used COMPASS data to evaluate ischemic stroke and ischemic stroke subtype variants identified in European-ancestry populations. ${ }^{6-8,10-12}$ In COMPASS, we tested the reported European-ancestry GWAS SNP (i.e. 'index SNP') as well as SNPs in moderate LD $\left(\mathrm{r}^{2} \searrow 0.50\right)$ with the index SNP $( \pm 500 \mathrm{kB})$ based on HapMap CEU to capture more broadly the European-ancestry 'index signal.' Again, we applied a Bonferronicorrected significance threshold.

\section{Power}

Using Quanto (v1.2.4), ${ }^{25}$ we estimated reasonable power ( $280 \%$ ) to detect low frequency common variants ( $0.09 \geq \mathrm{MAF} \unlhd \mathbf{} \mathbf{} .15)$ associated with $250 \%$ increased (or decreased) risk of stroke in our discovery aim. As MAF increased to 0.50 , we had reasonable power to detect increasingly smaller SNP-stroke associations, down to $\sim 25 \%$ increased (or decreased) risk (i.e. effect size $\approx \pm 0.22-0.29$ ). Power to detect rarer variants, or common variants with more modest associations, was limited. ${ }^{26}$

\section{RESULTS}

\section{Discovery of stroke-associated loci}

COMPASS comprises 14,745 African-Americans including 1,365 ischemic and 1,592 total stroke cases (Supplemental Table I). We identified an association between rs4471613 $(15 \mathrm{q} 21.3)$ and total stroke in African-Americans, [beta(SE) $=0.82(0.15), \mathrm{P}=3.9 \times 10^{-8}$ ] with suggestive evidence for ischemic stroke $\left[\right.$ beta(SE) $\left.=0.90(0.18), \mathrm{P}=4.6 \times 10^{-7}\right]$. An additional 18 SNPs had suggestive evidence of association $\left(\mathrm{P}<1 \times 10^{-6}\right)$ (Supplemental Table II). Five were for ischemic stroke-including PTPRG, CDC5L and HPS4-ASPHD2 loci SNPs and one intergenic SNP (chr14q31)-and 15 SNPs were for total stroke-including SNPs in the PTPRG, HDAC9 and WDFY4 loci and intergenic regions. Two SNPs, rs704341 (PTPRG/ intron) and rs4471613 (15q21.3/intergenic), were suggestively associated with both ischemic and total stroke. In sensitivity analyses including only the cohort studies, associations for both SNPs remained below the $\mathrm{P}<10^{-6}$ threshold (Supplemental Table III). 


\section{Validation of COMPASS SNPs in METASTROKE}

None of the 19 SNPs from COMPASS (or SNPs in LD with them) met the Bonferroni threshold for significance in METASTROKE, $\mathrm{P}=0.05 / 19=0.003$. We did observe nominal associations for 5q35.2 (rs4867766, $\mathrm{P}=0.031$ ) and 1p31.1 (rs35020936, $\mathrm{P}=0.018)$ (Table 1). In 5q35.2, the intergenic SNP rs4867766 was the top SNP in both race/ethnicities, despite different MAF, 0.08 in African-American and 0.21 in European-Ancestry populations whereas in 1p31.1, the top SNP differed in the populations (Table 1).

\section{Testing of Ischemic Stroke GWAS loci identified in European-ancestry populations}

None of the European-ancestry index SNPs were associated with ischemic stroke in AfricanAmericans at $\mathrm{P}=0.007$ ( $\mathrm{P}=0.05 / 7$ loci tested) (Table 2). When we also investigated SNPs in modest LD (Supplementary Table IV), we found suggestive evidence of association $(\mathrm{P}<0.05)$ for the PITX2, HDAC9, CDKN2A/CDKN2B and ZFHX3 loci.

\section{DISCUSSION}

The COMPASS collaboration represents the first large-scale GWAS meta-analysis of stroke in African-Americans. We report a novel genome-wide association for total stroke at the 15 q21.3 locus and report 14 additional loci suggestively associated with total or ischemic stroke in African-Americans. Additionally, in our African-American population, we found suggestive evidence of replication for the PITX2, HDAC9, CDKN2A/CDKN2B and ZFHX3 loci previously associated with stroke in European-Ancestry populations, pointing to potential shared mechanisms for stroke susceptibility.

The top SNP, rs4471613, is located near the $3^{\prime}$ region of the aquaporin 9 gene $(A Q P 9)$, the aldehyde dehydrogenase 1 family, member A2 (ALDH1A2) and hepatic lipase (LIPC) genes. Prior work suggests a role for $A Q P 9$ in cerebral energy metabolism as well as in brain ischemia, development of cerebral edema and post-ischemic reuptake of glycerol and lactate. ${ }^{27,} 28$ Intergenic SNPs in this region also are associated with blood lipids in populations of diverse ancestry ${ }^{29}$ and hypertension in African Americans. ${ }^{30}$ While this region is mechanistically appealing, rs4471613 is in low LD with these reported SNPs. The location of rs4471613 in a H3-lysine-27-acetylation histone mark in seven different cell types reflecting five tissues [ENCODE data from UCSC genome browser (https:// genome.ucsc.edu)] suggests a possible regulatory function for this SNP, or neighboring SNPs in this region. However, this SNP was not significantly associated with ischemic stroke in METASTROKE $(\mathrm{P}=0.13)$. Further evaluation of genetic factors influencing stroke across the $A Q P 9-L I P C$ region, particularly in populations of African-descent, is warranted.

Two intergenic loci (5q35 and 1p31.1) identified in COMPASS were modestly associated $(\mathrm{P}<0.05)$ with stroke in METASTROKE. Overall, the lack of strong replication in the larger METASTROKE European-ancestry population urges caution when interpreting COMPASS associations. However, as has been reported for other phenotypes, ${ }^{31}$ COMPASS loci could be unique to African-ancestry populations. Of note, the HDAC9 variants associated with large vessel stroke in European-ancestry populations ${ }^{7,12}$ are located more than $500 \mathrm{~kb}$ from the novel intronic HDAC9 variant identified in COMPASS. 
Replication of stroke GWAS variants across different ancestry/ethnicity groups are lacking but important for prioritizing genetic variants for translational research and understanding population differences in stroke burden. In our secondary aim to replicate prior GWAS findings from European-ancestry populations, we found suggestive evidence of replication $(\mathrm{P}<0.05)$ for four loci. Interestingly, four of the six nominally significant SNPs in these loci were originally associated with cardioembolic stroke in European-ancestry populations. None were significant after Bonferroni correction; nonetheless, these trends suggest that variants in these loci may influence stroke risk independent of race/ethnicity.

Some limitations of our study deserve mention. COMPASS case-control studies are limited to non-fatal and less severe strokes than longitudinal cohort studies, and are more likely to suffer from potential selection bias. However, findings from analyses of the cohort studies were generally similar to the overall meta-analysis, though a rare intronic variant in $C H D 3$, rs9899375, reached significance, $\mathrm{P}=5.2 \times 1^{-9}$, in the cohort analyses only.

The limited number of African-American stroke samples in COMPASS restricts power to discover new loci and also to replicate previous associations of modest effect size.

Additionally, SNP imputation in admixed populations, such as African Americans, is challenging due to differing LD patterns and greater genetic diversity than in the standard reference panels. Given the use of YRI/CEU HapMap II reference panels rather than African American-specific panels for imputation, we may have slightly reduced power to detect associations (resulting in greater chance of false negatives), and reduced ability to localize stroke-associated variants in our population.

Total and ischemic stroke are heterogeneous outcomes. Recent GWAS of ischemic stroke have detected heterogeneity of associations across stroke subtypes. ${ }^{7}$ Although the largest GWAS of stroke in African-Americans, COMPASS still lacks adequate numbers to stratify by stroke subtype. In COMPASS studies with subtype information, ischemic stroke is predominant and lacunar (or small vessel) stroke is the most common ischemic stroke subtype, consistent with data indicating that intracranial atherosclerotic and lacunar (or small vessel) strokes predominate in African-Americans. ${ }^{8,} 14$ Furthermore, small vessel ischemic stroke incidence in African-Americans is more than double that of US whites. ${ }^{33}$ Thus, some of the observed differences in risk loci may reflect differences in distributions of major ischemic stroke subtypes across diverse populations. However, these differences offer an opportunity to identify shared or distinct risk factors and mechanisms for specific ischemic stroke subtypes across diverse populations, such as the nominally significant replication of cardioembolic-associated loci (PITX2, ZFHX3) in COMPASS. Replication of African-American GWAS SNPs in a European-ancestry population is not ideal due to differences in population LD, with African-ancestry populations having higher genetic diversity on average than European populations. ${ }^{34}$ Yet, when we broadly interrogated the 'index SNP' signals, we observed modest replication. Such data may help localize the actual causal variants within GWAS signals. 


\section{SUMMARY}

Despite its limitations, our study presents results for a unique, lesser-studied population with a substantial stroke burden. Published stroke GWAS have identified only a handful of replicable associations, perhaps reflecting stroke phenotypic complexity and potential geneby-environment influences in different populations. ${ }^{35}$ Thus, genetic association studies in populations of diverse ancestry, such as this one, are critical to understanding the genetic basis of stroke, an increasingly important global disease.

\section{Supplementary Material}

Refer to Web version on PubMed Central for supplementary material.

\section{Acknowledgments}

The authors thank the staff and participants of the ARIC study for their important contributions; the WHI investigators and staff for their dedication, and the study participants for making the program possible. For a list of investigators who have contributed to WHI science, please visit: https://www.whi.org/researchers/Documents $\% 20 \% 20$ Write $\% 20$ a \%20Paper/WHI\%20Investigator\%20Long\%20List.pdf)

\section{FUNDING}

ARIC Study Supported by National Heart, Lung, and Blood Institute contracts (HHSN268201100005C, HHSN268201100006C, HHSN268201100007C, HHSN268201100008C, HHSN268201100009C, HHSN268201100010C, HHSN268201100011C, and HHSN268201100012C), R01HL087641, R01HL59367 and R01HL086694; National Human Genome Research Institute contract U01HG004402; and NIH contract HHSN268200625226C. Infrastructure was partly supported by Grant Number UL1RR025005, a component of the NIH and NIH Roadmap for Medical Research.

CHS Supported by National Heart, Lung, and Blood Institute contracts HHSN268201200036C, HHSN268200800007C, N01HC55222, N01HC85079, N01HC85080, N01HC85081, N01HC85082, N01HC85083, N01HC85086; and grants U01HL080295, R01HL087652, R01HL105756, R01HL103612, and R01HL120393 with contributions from the National Institute of Neurological Disorders and Stroke. Additional support was provided through R01AG023629 from the National Institute on Aging. A full list of principal CHS investigators and institutions can be found at CHS-NHLBI.org. The provision of genotyping data was supported in part by the National Center for Advancing Translational Sciences, grant UL1TR000124, and the National Institute of Diabetes and Digestive and Kidney Disease Research Center grant DK063491 to the Southern California Diabetes Endocrinology Research Center. The content is solely the responsibility of the authors and does not necessarily represent the official views of the NIH.

GEOS Supported by the NIH Genes, Environment and Health Initiative Grant U01 HG004436, as part of the GENEVA consortium, with additional support provided by the Mid-Atlantic Nutrition and Obesity Research Center (P30-DK072488); the Office of Research and Development, Medical Research Service, and the Baltimore Geriatrics Research, Education, and Clinical Center of the Department of Veterans' Affairs. Study recruitment and datasets assembly were supported by a Cooperative Agreement with the Division of Adult and Community Health, Centers for Disease Control and grants from the National Institute of Neurological Disorders and Stroke and the NIH Office of Research on Women's Health (R01-NS45012, U01-NS069208-01). Y.Cheng was supported by the Department of Veterans' Affairs career development award (1IK2BX001823).

HANDLS Supported by the Intramural Research Program of the NIH, National Institute of Aging and the National Center on Minority Health and Health Disparities (project\# Z01-AG000513 and human subjects protocol\# 2009-149).

ISGS and SWISS ISGS and SWISS were supported by the National Institute of Neurological Disorders and Stroke grants (R01 NS42733; PI Meschia) and (R01NS39987; PI Meschia), respectively with additional support, in part, from the Intramural Research Program of the National Institute of Aging (Z01 AG000954-06; PI Singleton). Both studies used samples and clinical data from the NIH-NINDS Human Genetics Resource Center DNA and Cell Line Repository, human subjects protocol\# 2003-081 and 2004-147. 
VISP Funded by the National Institute of Neurological Disorders and Stroke (R01-NS34447). GWAS data for a subset of VISP participants supported by the National Human Genome Research Institute (U01-HG005160), as part of the Genomics and Randomized Trials Network (PI: Sale and Worrall).

WHI Supported by the National Heart, Lung, and Blood Institute, NIH, and Department of Health and Human Services through contracts N01WH22110, 24152, 32100-2, 32105-6, 32108-9, 32111-13, 32115, 32118-32119, 32122 , 42107-26, 42129-32, and 44221. Funders had no role in study design, data collection and analysis, decision to publish, or manuscript preparation.

\section{References}

1. Roger VL, Go AS, Lloyd-Jones DM, Benjamin EJ, Berry JD, Borden WB, et al. Heart disease and stroke statistics-2012 update: a report from the American Heart Association. Circulation. 2012; 125:e2-e220. [PubMed: 22179539]

2. Saeed M. Editorial comment-Unraveling the pagodian architecture of stroke as a complex disorder. Stroke. 2004; 35:824-825. [PubMed: 15044810]

3. Hajat C, Dundas R, Stewart JA, Lawrence E, Rudd AG, Howard R, et al. Cerebrovascular risk factors and stroke subtypes: differences between ethnic groups. Stroke. 2001; 32:37-42. [PubMed: 11136911]

4. Liao D, Myers R, Hunt S, Shahar E, Paton C, Burke G, et al. Familial history of stroke and stroke risk. The Family Heart Study. Stroke. 1997; 28:1908-1912. [PubMed: 9341694]

5. Bak S, Gaist D, Sindrup SH, Skytthe A, Christensen K. Genetic liability in stroke: a long-term follow-up study of Danish twins. Stroke. 2002; 33:769-774. [PubMed: 11872902]

6. Gretarsdottir S, Thorleifsson G, Manolescu A, Styrkarsdottir U, Helgadottir A, Gschwendtner A, et al. Risk variants for atrial fibrillation on chromosome $4 \mathrm{q} 25$ associate with ischemic stroke. Ann Neurol. 2008; 64:402-409. [PubMed: 18991354]

7. Bellenguez C, Bevan S, Gschwendtner A, Spencer CC, Burgess AI, Pirinen M, et al. Genome-wide association study identifies a variant in HDAC9 associated with large vessel ischemic stroke. Nature genetics. 2012; 44:328-333. [PubMed: 22306652]

8. Holliday EG, Maguire JM, Evans TJ, Koblar SA, Jannes J, Sturm JW, et al. Common variants at 6p21.1 are associated with large artery atherosclerotic stroke. Nature genetics. 2012; 44:1147-1151. [PubMed: 22941190]

9. Dichgans M, Malik R, Konig IR, Rosand J, Clarke R, Gretarsdottir S, et al. Shared genetic susceptibility to ischemic stroke and coronary artery disease: a genome-wide analysis of common variants. Stroke. 2014; 45:24-36. [PubMed: 24262325]

10. Ikram MA, Seshadri S, Bis JC, Fornage M, Destefano AL, Aulchenko YS, et al. Genomewide association studies of stroke. N Engl J Med. 2009; 360:1718-1728. [PubMed: 19369658]

11. Kilarski LL, Achterberg S, Devan WJ, Traylor M, Malik R, Lindgren A, et al. Meta-analysis in more than 17,900 cases of ischemic stroke reveals a novel association at $12 \mathrm{q} 24.12$. Neurology. 2014; 83:678-685. [PubMed: 25031287]

12. Traylor M, Farrall M, Holliday EG, Sudlow C, Hopewell JC, Cheng YC, et al. Genetic risk factors for ischaemic stroke and its subtypes (the METASTROKE collaboration): a meta-analysis of genome-wide association studies. The Lancet Neurology. 2012; 11:951-962. [PubMed: 23041239]

13. Lloyd-Jones D, Adams RJ, Brown TM, Carnethon M, Dai S, De Simone G, et al. Heart disease and stroke statistics-2010 update: a report from the American Heart Association. Circulation. 2010; 121:e46-e215. [PubMed: 20019324]

14. White H, Boden-Albala B, Wang C, Elkind MS, Rundek T, Wright CB, et al. Ischemic stroke subtype incidence among whites, blacks, and Hispanics: the Northern Manhattan Study. Circulation. 2005; 111:1327-1331. [PubMed: 15769776]

15. The ARIC Investigators. The Atherosclerosis Risk in Communities (ARIC) Study: design and objectives. Am J Epidemiol. 1989; 129:687-702. [PubMed: 2646917]

16. Fried LP, Borhani NO, Enright P, Furberg CD, Gardin JM, Kronmal RA, et al. The Cardiovascular Health Study: design and rationale. Ann Epidemiol. 1991; 1:263-276. [PubMed: 1669507] 
17. Wassel Fyr CL, Kanaya AM, Cummings SR, Reich D, Hsueh WC, Reiner AP, et al. Genetic admixture, adipocytokines, and adiposity in Black Americans: the Health, Aging, and Body Composition study. Human genetics. 2007; 121:615-624. [PubMed: 17390149]

18. Women's Health Initiative Study Group. Design of the Women's Health Initiative clinical trial and observational study. Control Clin Trials. 1998; 19:61-109. [PubMed: 9492970]

19. Cheng YC, O'Connell JR, Cole JW, Stine OC, Dueker N, McArdle PF, et al. Genome-wide association analysis of ischemic stroke in young adults. G3 (Bethesda). 2011; 1:505-514. [PubMed: 22384361]

20. Meschia JF, Brott TG, Brown RD Jr, Crook RJ, Frankel M, Hardy J, et al. The Ischemic Stroke Genetics Study (ISGS) Protocol. BMC neurology. 2003; 3:4. [PubMed: 12848902]

21. Spence JD, Howard VJ, Chambless LE, Malinow MR, Pettigrew LC, Stampfer M, et al. Vitamin Intervention for Stroke Prevention (VISP) trial: rationale and design. Neuroepidemiology. 2001; 20:16-25. [PubMed: 11174041]

22. Evans MK, Lepkowski JM, Powe NR, LaVeist T, Kuczmarski MF, Zonderman AB. Healthy aging in neighborhoods of diversity across the life span (HANDLS): overcoming barriers to implementing a longitudinal, epidemiologic, urban study of health, race, and socioeconomic status. Ethn dis. 2010; 20:267-275. [PubMed: 20828101]

23. Meschia JF, Brown RD Jr, Brott TG, Chukwudelunzu FE, Hardy J, Rich SS. The Siblings With Ischemic Stroke Study (SWISS) protocol. BMC Med Genet. 2002; 3:1. [PubMed: 11882254]

24. Willer CJ, Li Y, Abecasis GR. METAL: fast and efficient meta-analysis of genomewide association scans. Bioinformatics. 2010; 26:2190-2191. [PubMed: 20616382]

25. Gauderman, WJ.; Morrison, JM. QUANTO 1.1: A computer program for power and sample size calculations for genetic-epidemiology studies. 2006. http://hydra.usc.edu/gxe

26. Gschwendtner A, Bevan S, Cole JW, Plourde A, Matarin M, Ross-Adams H, et al. Sequence variants on chromosome 9p21.3 confer risk for atherosclerotic stroke. Ann Neurol. 2009; 65:531539. [PubMed: 19475673]

27. Lettre G, Palmer CD, Young T, Ejebe KG, Allayee H, Benjamin EJ, et al. Genome-wide association study of coronary heart disease and its risk factors in 8,090 African Americans: the NHLBI CARe Project. PLoS genetics. 2011; 7:e1001300. [PubMed: 21347282]

28. Badaut J. Aquaglyceroporin 9 in brain pathologies. Neuroscience. 2010; 168:1047-1057. [PubMed: 19850108]

29. Teslovich TM, Musunuru K, Smith AV, Edmondson AC, Stylianou IM, Koseki M, et al. Biological, clinical and population relevance of 95 loci for blood lipids. Nature. 2010; 466:707713. [PubMed: 20686565]

30. Adeyemo A, Gerry N, Chen G, Herbert A, Doumatey A, Huang H, et al. A genome-wide association study of hypertension and blood pressure in African Americans. PLoS Genet. 2009; 5:e1000564. [PubMed: 19609347]

31. Li J, Glessner JT, Zhang H, Hou C, Wei Z, Bradfield JP, et al. GWAS of blood cell traits identifies novel associated loci and epistatic interactions in Caucasian and African-American children. Hum Mol Genet. 2013; 22:1457-1464. [PubMed: 23263863]

32. Hao K, Chudin E, McElwee J, Schadt EE. Accuracy of genome-wide imputation of untyped markers and impacts on statistical power for association studies. BMC Genet. 2009; 10:27. [PubMed: 19531258]

33. Woo D, Gebel J, Miller R, Kothari R, Brott T, Khoury J, et al. Incidence rates of first-ever ischemic stroke subtypes among blacks: a population-based study. Stroke. 1999; 30:2517-2522. [PubMed: 10582971]

34. Altshuler DM, Gibbs RA, Peltonen L, Dermitzakis E, Schaffner SF, Yu F, et al. Integrating common and rare genetic variation in diverse human populations. Nature. 2010; 467:52-58. [PubMed: 20811451]

35. Dichgans M, Markus HS. Genetic association studies in stroke: methodological issues and proposed standard criteria. Stroke. 2005; 36:2027-2031. [PubMed: 16051898] 


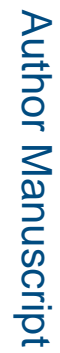

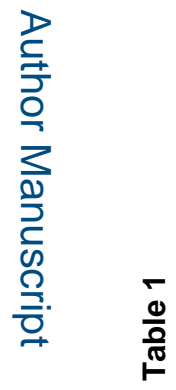

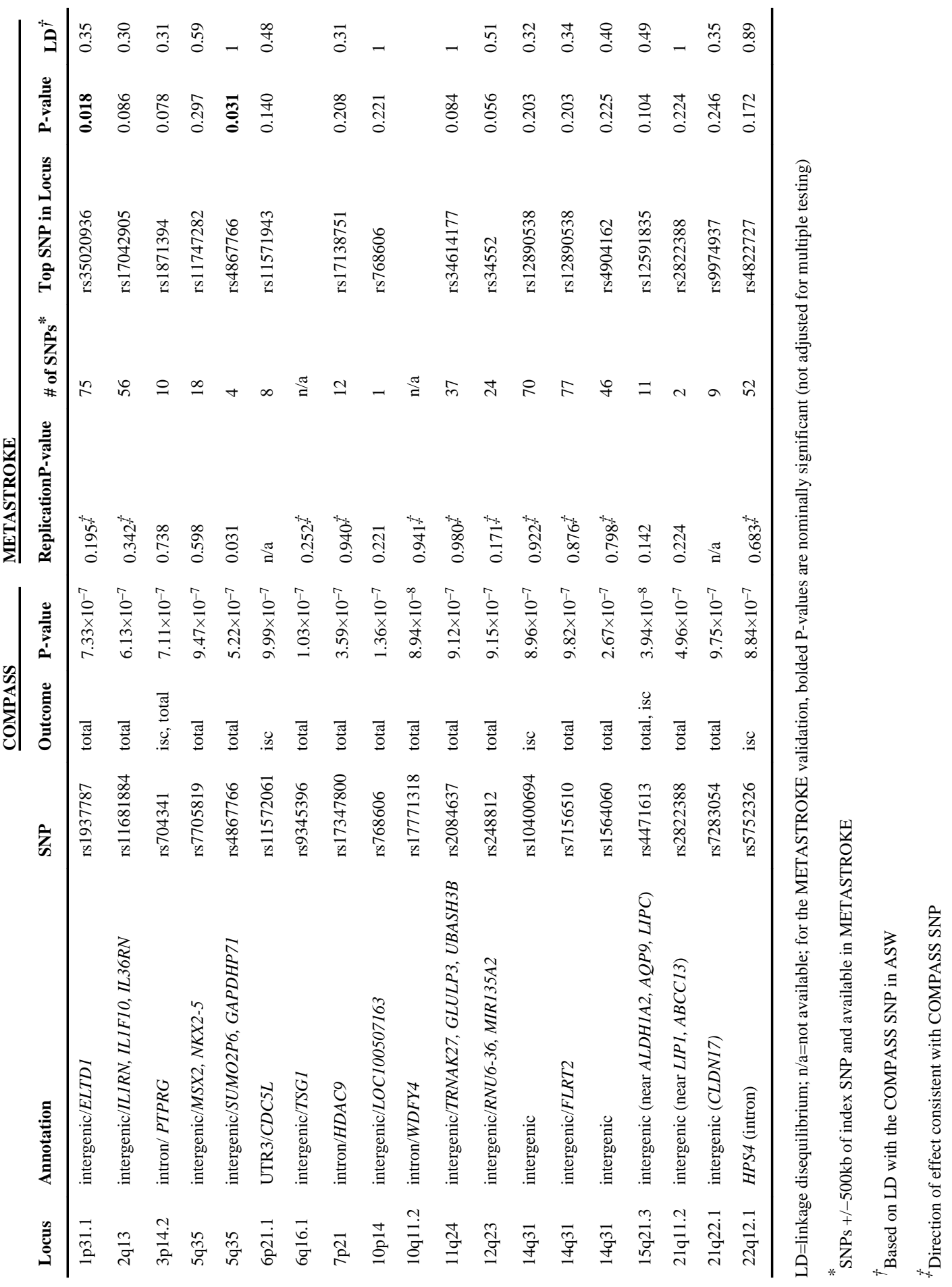

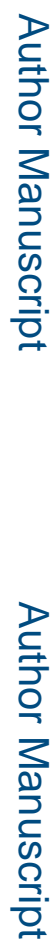

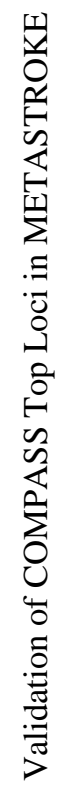




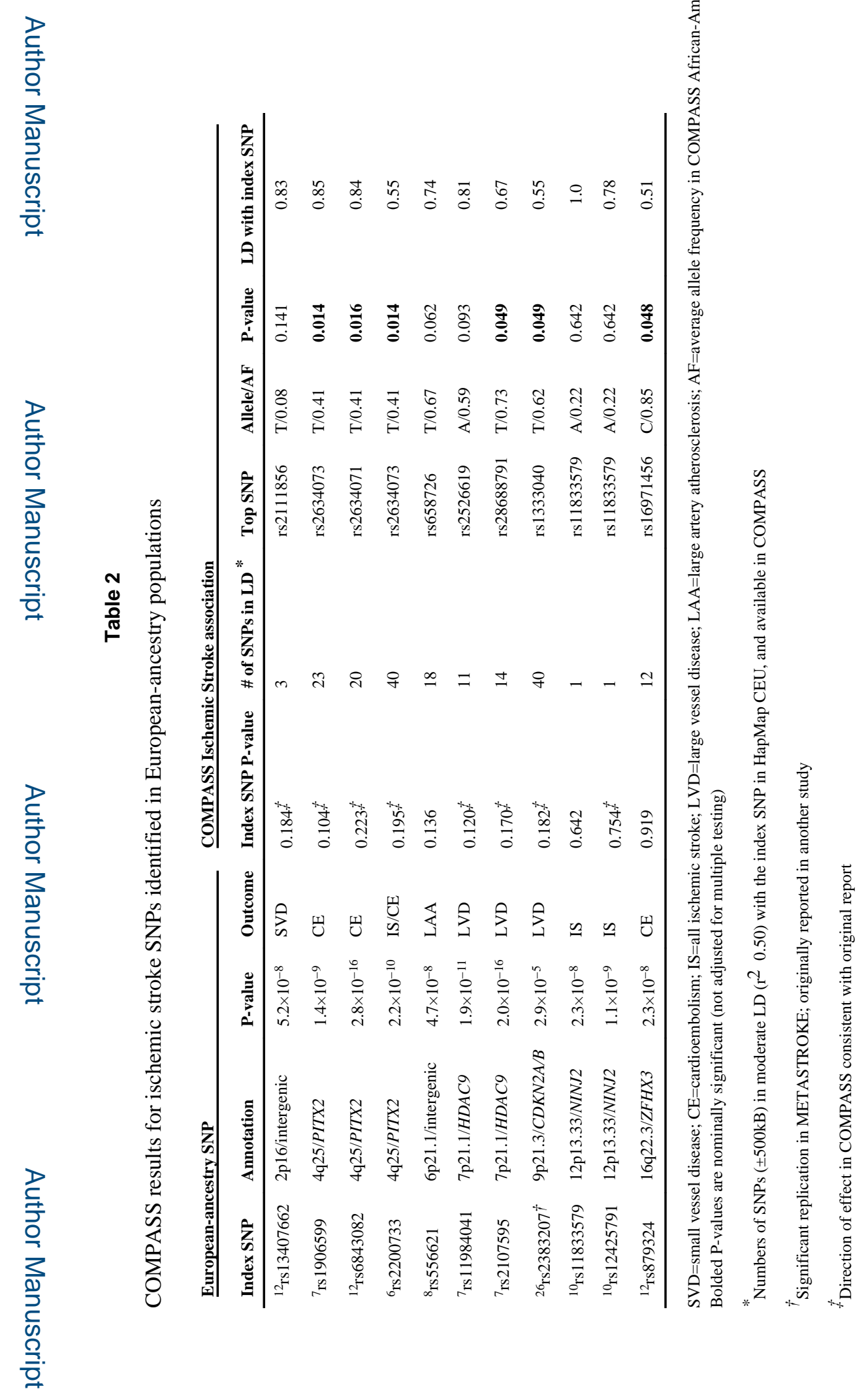

Stroke. Author manuscript; available in PMC 2016 August 01. 